\title{
Management of Skeletal Class-II Malocclusion Using Advansync 2: A Case Series
}

\author{
Abinaya Somaskandhan', Krishna Priyaa Suresh ${ }^{1}$, \\ Srinivasan Boovaraghavan ${ }^{2}$, Priya R. ${ }^{1}$, Devaki Vijayalakshmi ${ }^{3}$ \\ ${ }^{1}$ Postgraduate student, Dept of Orthodontics and Dentofacial Orthopaedics, Meenakshi Ammal Dental College, \\ Mugalivakkam, Porur, Chennai- 600125, ' Reader, Dept of Orthodontics and Dentofacial Orthopaedics, Meenakshi \\ Ammal Dental College. Chennai, ${ }^{3}$ Head of the Department, Dept of Orthodontics and Dentofacial Orthopaedics, \\ Meenakshi Ammal Dental College, Chennai
}

\begin{abstract}
Angle's class II division 1 malocclusion, being the most commonly occuring type of malocclusion, is challenging to treat especially when there is an underlying skeletal discrepancy. Various fixed functional appliances have been developed over the years by different authors for correction of skeletal class II malocclusion due to retrognathic mandible. Among the fixed functional appliances available for class II correction, Advansync2 (Ormco Co, Glendora, Calif) also known as molar to molar appliance has been used effectively in the recent past. It is a fixed tooth-born functional appliance with an advantage of allowing concurrent use of fixed orthodontic therapy. This facilitates reduction of the overall treatment duration. Two case reports of patients treated using the Advansync 2 class II corrector has been presented along with the skeletal and dentoalveolar changes observed during the treatment. The effects observed were similar to most fixed functional appliances however the treatment duration was considerably reduced in all the cases.
\end{abstract}

Keywords: Class II, Advansync2, fixed functional appliance, cephalometrics.

\section{Introduction:}

Angle's class II malocclusion is one of the most common problems affecting one third of the population and it has been a challenge for all the orthodontists owing to its difficulty in predicting its aetiology and its variable nature of representation ${ }^{[1]}$. In Indian population, orthodontists face a lot of class II div 1 cases mostly characterized by anteroposterior dental discrepancy which becomes severe when combined with an underlying skeletal disharmony. Although class II malocclusions can be associated with mandibular deficiency or maxillary protrusion or combination,

\section{Corresponding Author:}

\section{Dr. Abinaya Somaskandhan}

Postgraduate Student, Dept. of Orthodontics and Dentofacial Orthopaedics, Meenakshi Ammal Dental College, Mugalivakkam, Porur, Chennai- 600125 e-mail: abi.kandhan@gmail.com aprevalence of $14.6 \%$ among $10-13 y$ ears is found for mandibular retrusion ${ }^{[2]}$.

The treatment modality should consider factors such as aetiology, age of the patient, severity of malocclusion, growth status, patient compliance etc. for the proper selection of the appliance ${ }^{[2,3]}$. In growing population,Class II elastics or removable functional appliance are usually advocated for class II malocclusion. However, patient compliance becomes a major concern to employ these treatment modalities.

Fixed functional appliances were developed to bring the mandible forward regardless of patient compliance. Use of fixed functional appliance is an effective way to treat Class IImalocclusion in post adolescent patients where the growth is mostly completed, thus dentoalveolar changes takes place predominantly rather than skeletal changes ${ }^{[4]}$. The Herbst appliance developed by H.Pancherz has been used routinely for nearly 40 years ${ }^{[5]}$. Over the years there have been many design variations developed by different authors in order to 
increase the efficiency and reduce the patient discomfort during appliance wear.

AdvanSync2 is a fixed tooth borne functional appliance developed by Terry Dischinger in 2008 . It facilitates functional therapy along with fixed mechanotherapy to reduce the treatment duration and also to improve compliance of the patient ${ }^{[6]}$. It supposedly gives greater skeletal change when compared to other similar fixed functional appliances.
This article consists of a series of two cases whose primary treatment plan for fulfilling the treatment objectives was an orthodontic-fixed functional combined approach. Cephalometric parameters revealed orthognathic maxilla and retrognathic mandible. Hence, mandibular advancement using Advansync2 appliance was planned for both the cases. The amount of skeletal and dental changes achieved at the end of the treatment have also been enumerated.

\section{Case 1:}

\section{Diagnosis and treatment plan:}
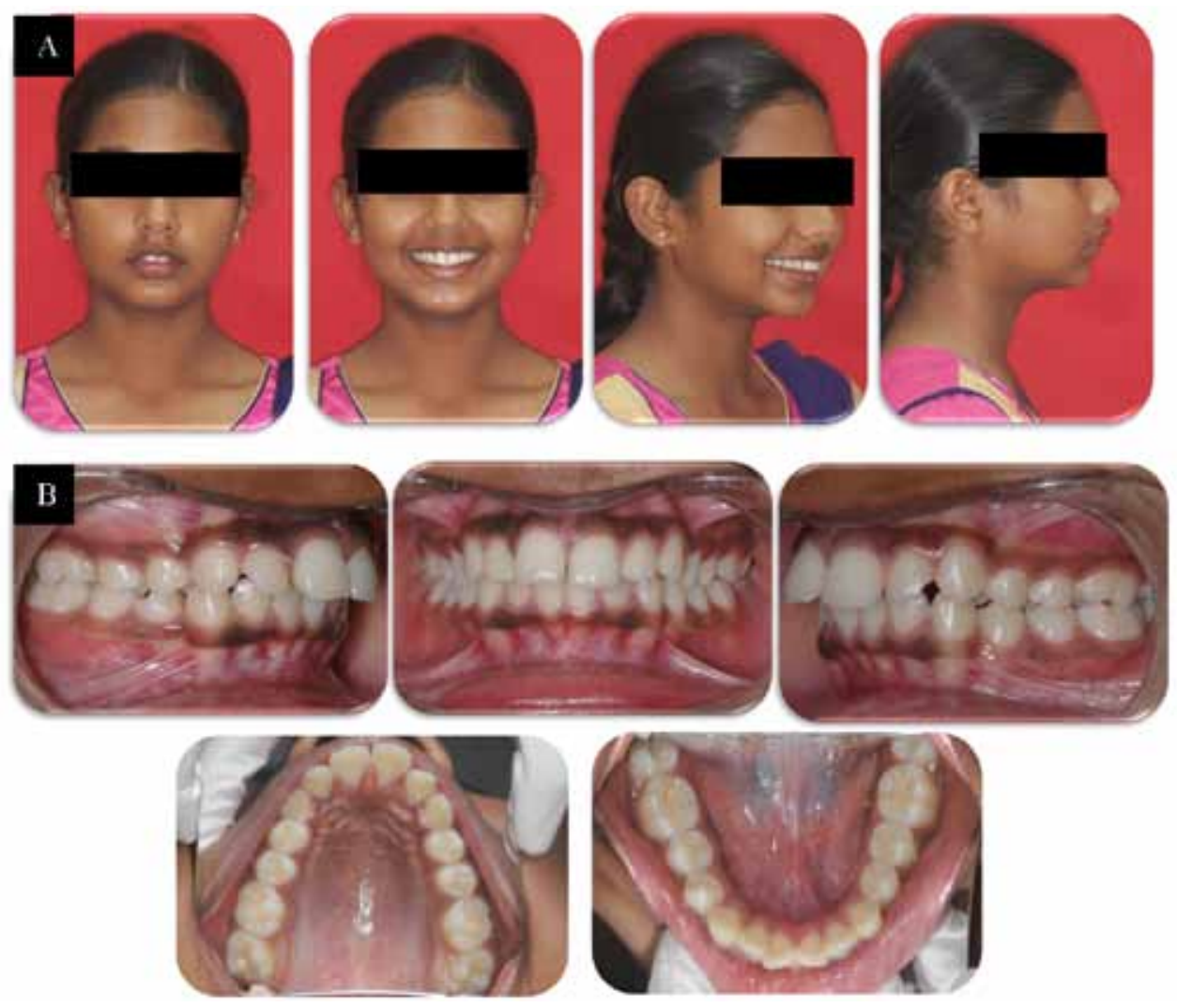

Figure 1: Pre-treatment A) Extra-oral B) Intra-oral photographs

A 15 year old female patient reported with a chief complaint of forwardly placed upper front teeth. She did not have any associated medical history and no associated habits. On extra-oral examinaton, she had a mesocephalic head type, mesomorphic body type with mesoprosopic facial pattern and an aesthetic built. Her profile examination revealed convex profile, posterior divergence, average nasolabial angle and average clinical FMA with a positive VTO (Figure 1A). On intra-oral examination, she had a bilateral class II molar and class
II canine relationship with an increased overjet of $8 \mathrm{~mm}$ and an overbite of $5 \mathrm{~mm}$. She had a tapered maxillary arch and ovoid mandibular arch with imbrications in lower anteriors. She had a decreased intercanine, interpremolar and intermolar width in the maxillary and mandibular $\operatorname{arch}$ (Figure 1B).

Cephalometric analysis revealed that the patient was in CVMI stage 3 which indicates that $25 \%-65 \%$ of the growth was remaining (Figure 2A). Accoding to Moyers 
classification of skeletal malocclusion, the patient had a horizontal type F (mandibular retrognathism) pattern; salzmann classification of skeletal class 2 division 1 and stage 8 of fishmans classification (Figure 2B). She had anorthognathic maxilla and retrognathic mandible, with average mandibular plane angle and a decreased lower anterior facial height.
Pre treatment orthopantomogram (OPG) revealed that the patient had full permanent dentition with no missing or supernumerary teeth (Figure 2C).

Based on the above findings, the patient was diagnosed with Angle's class II div 1 malocclusion on a class II skeletal base with orthognathic maxilla and retrognathic mandible on an average mandibular plane angle with proclined upper and lower incisors with an increased overjet of $8 \mathrm{~mm}$.
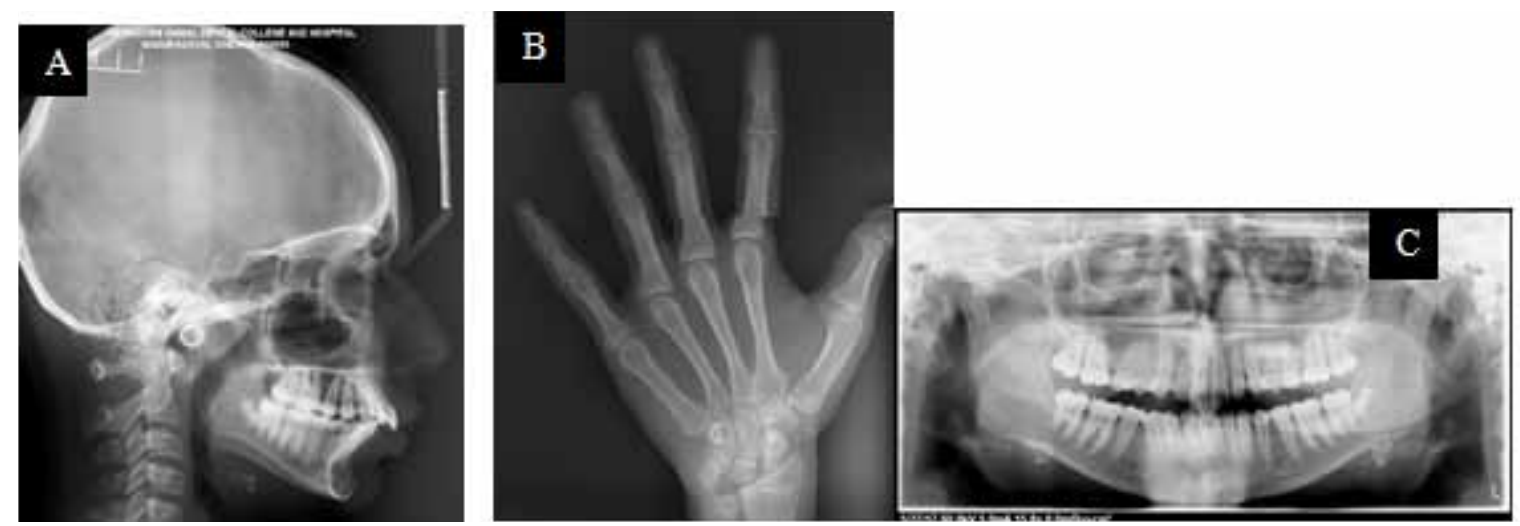

Figure 2: Pre-treatment radiographs A) Lateral cephalogram B) Hand wrist C) OPG

Treatment Progress: The advansync2 appliance was assembled initially (Figure 3) and activated for $3 \mathrm{~mm}$ thrice during the therapy. A full orthodontic appliance of 0.022 slot MBT system was bonded in the upper arch and the lower arch. Aligning and levelling was done sequentially with $0.016 \mathrm{NiTi}$ and $16 \times 22 \mathrm{NiTi}$. At the end of the fixed functional therapy, bilateral class I molar and class I canine was obtained (Figure 4). The archwires were in $17 \mathrm{X} 25 \mathrm{NiTi}$ at the end of the functional therapy.

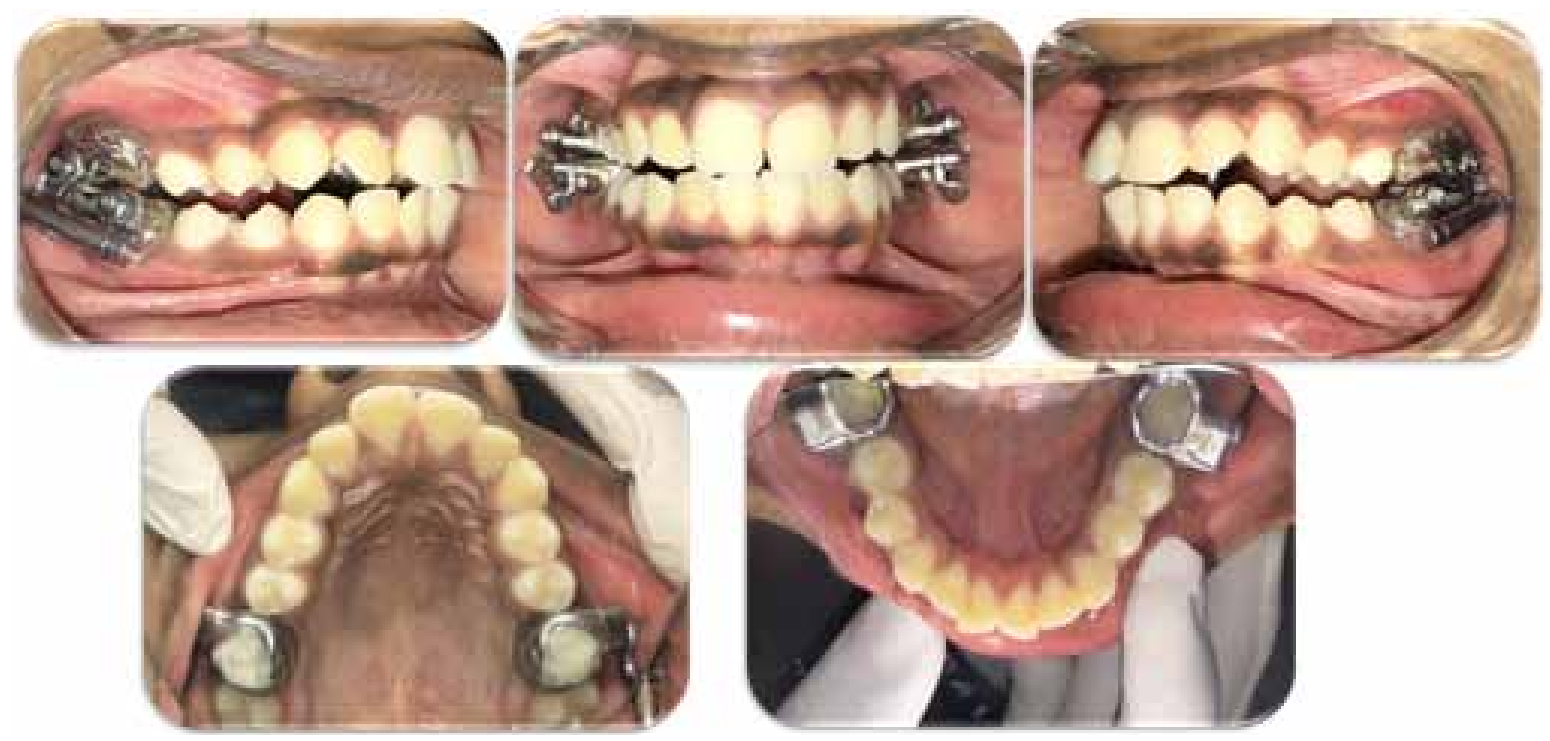

Figure 3: Advansync 2 Fixation 


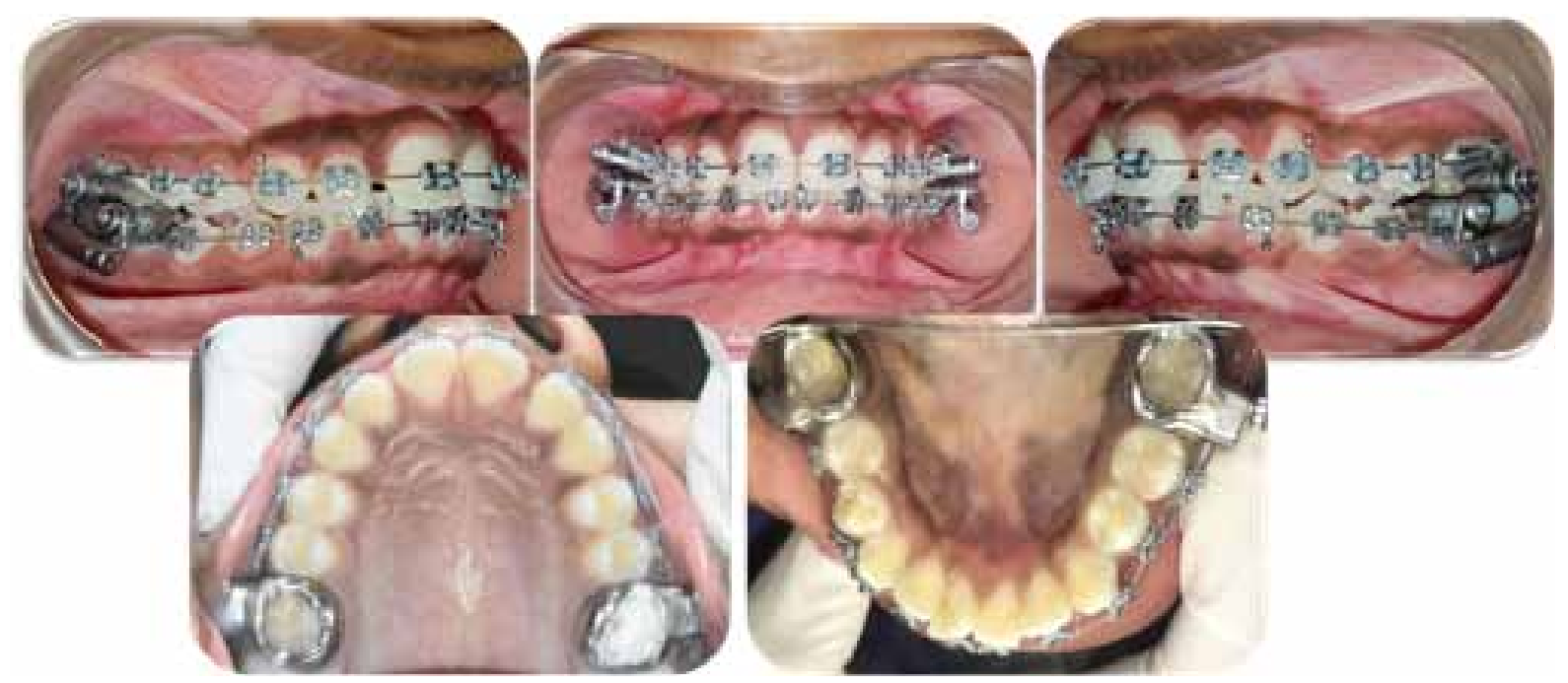

Figure 4: Post-functional photographs

Treatment Results: An overall increase in the maxillary inter-canine, inter-premolar and inter-molar widths of about $4 \mathrm{~mm}$ was achieved at the end of the treatment. The post treatment photographs indicate that the patient had obtained a very pleasing smile with an ideal class I molar and class I canine relationship with an ideal overjet and overbite (Figure 5). The radiographs imply that an ideal crown and root inclinations and angulations have been obtained along with funtional occlusion (Figure 6). The total duration of the treatment was 18 months. Beggs wrap around retainer was delivered for the upper arch and a bonded lingual retainer was fixed in the lower arch.
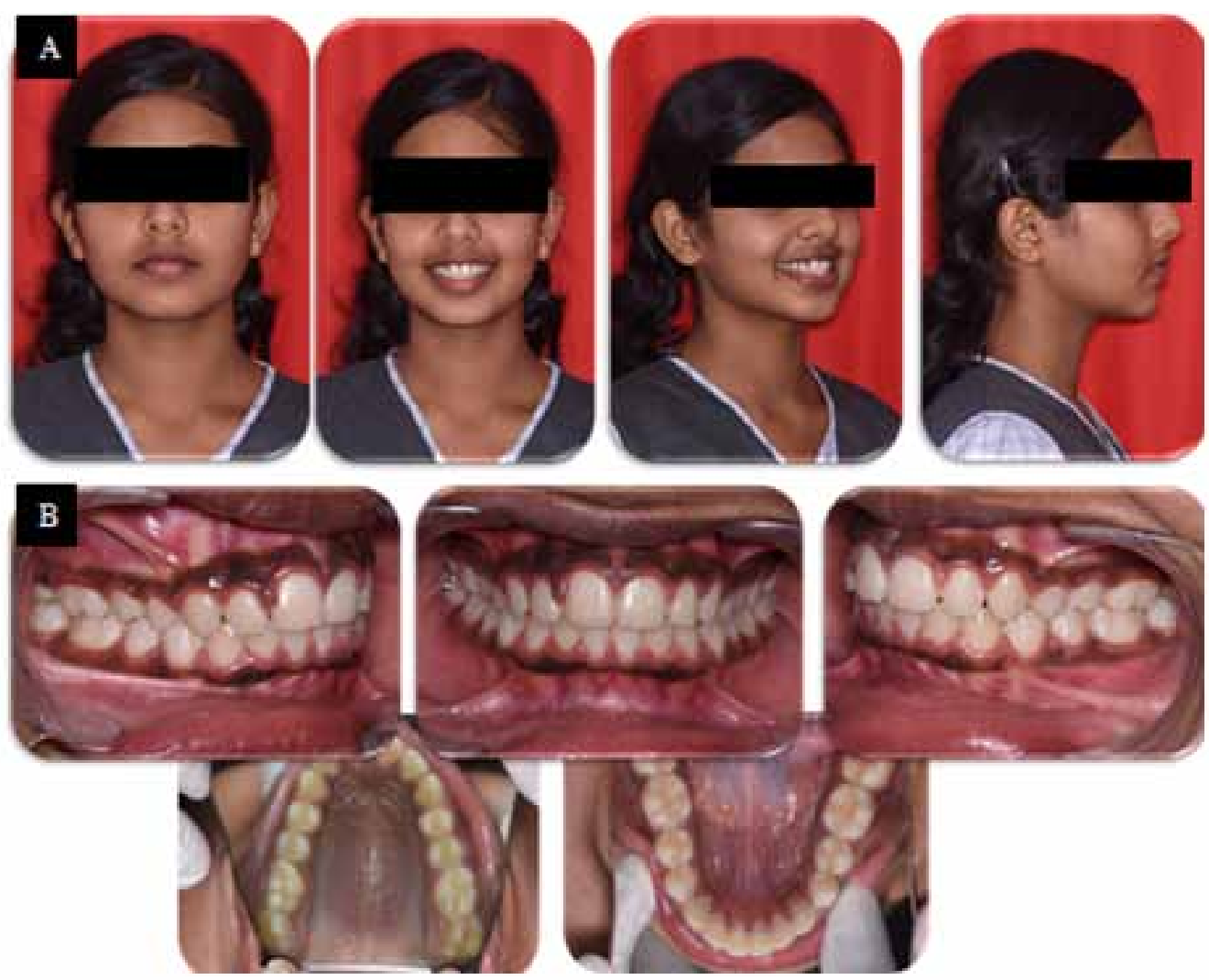

Figure 5: Post-treatment A) Extra-oral B) Intra-oral photographs 

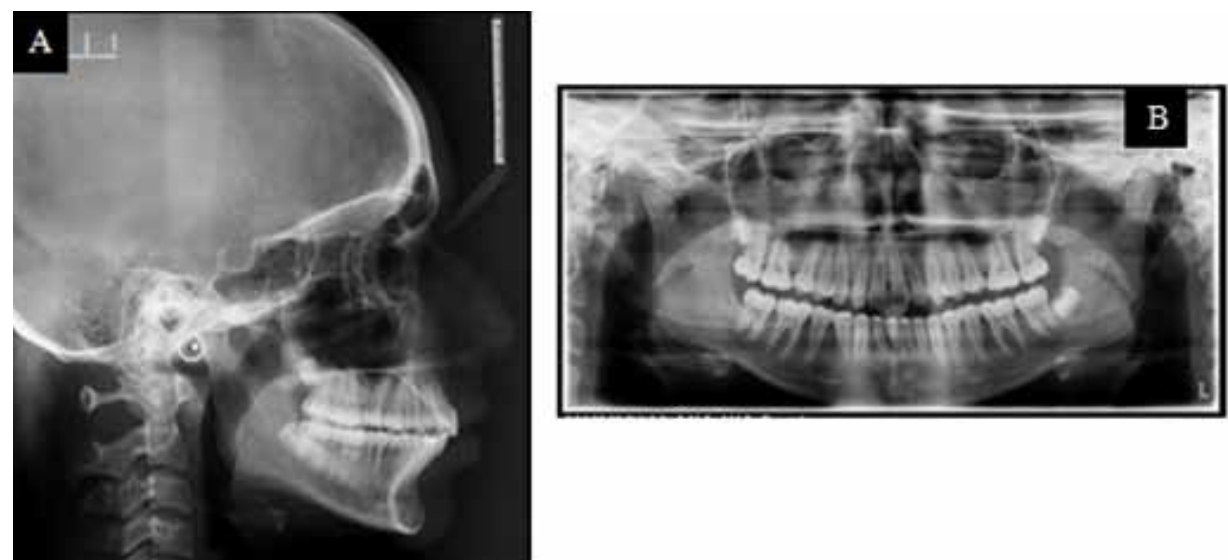

Figure 6: Post-treatment radiographs A) Lateral cephalogram B) OPG

\section{Case 2:}

Diagnosis and treatment plan: A 12 year old male patient reported to the Department of Orthodontics and Dentofacial Orthopaedics with a chief complaint of forwardly placed upper from teeth. No history of medical illness or trauma was elicited from the patient. There was no association of any temporo-mandibular joint disorders and no associated habits. On extra-oral examination the patient exhibited hypodivergent growth pattern with mesocephalic head type, mesomorphic body type and mesoprosopic facial form. Upon examination of his profile, he had a convex profile with posterior divergence with average nasolabial angle on a low clinical FMA. He exhibited a positive VTO (Figure 7A).

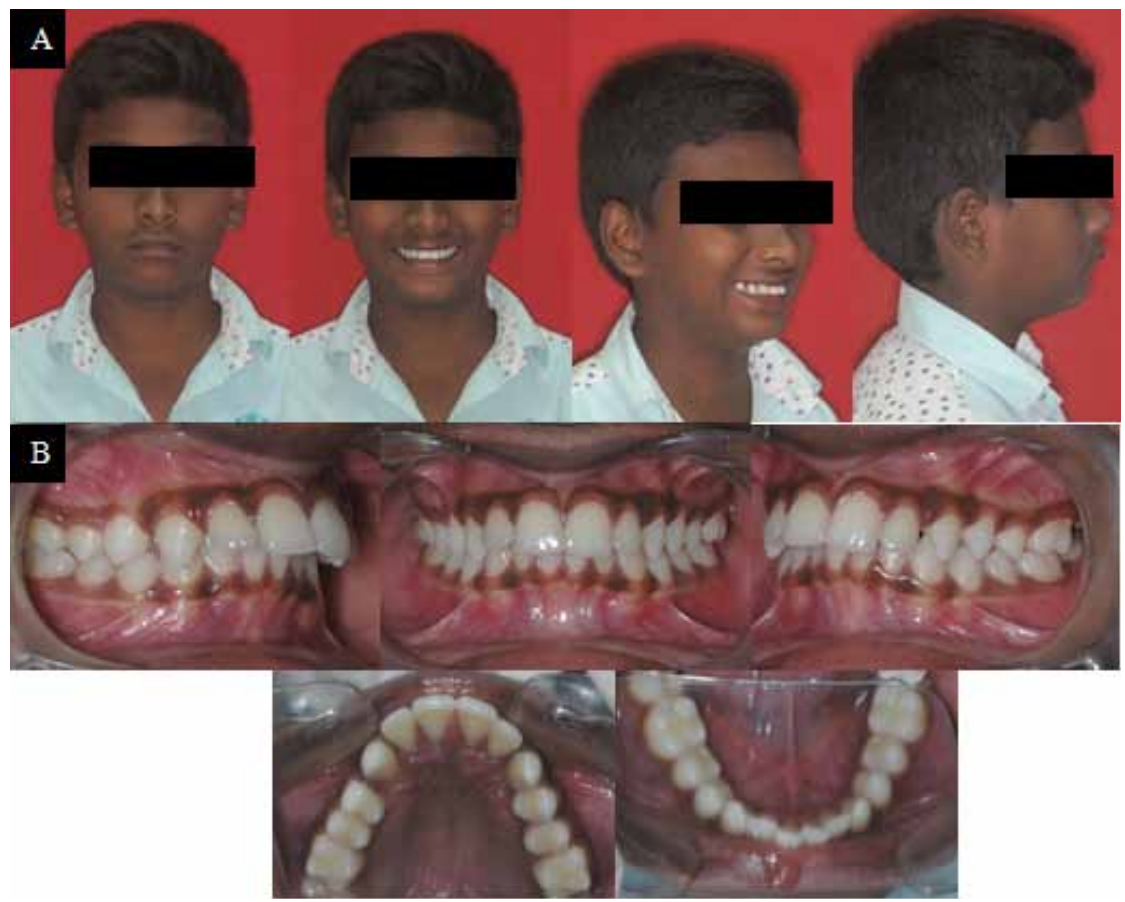

Figure 7: Pre-treatment A) Extra-oral B) Intra-oral photographs

On intra-oral examination, he had a bilateral endon molar relationship with bilateral end-on canine relationship. He had an increased overjet of $10 \mathrm{~mm}$ with increased overbite of $4 \mathrm{~mm}$. He had a tapered upper arch which was asymmetrical with spacing in relation to $12,13,14,22,23$. He had a decreased inter-canine width with average inter-premolar and inter-molar width in the maxillary dental arch. His mandibular arch was 
ovoid in shape with anterior crowding and decreased inter-canine, inter-premolar and inter-molar width (Figure 7B). On cephalometric analysis, the patient was in CVMI stage 3 which reveals that $25-65 \%$ of the growth was remaining (Figure 8A). Accoding to Moyers classification of skeletal malocclusion, the patient had a horizontal type $\mathrm{F}$ (mandibular retrognathism) pattern and salzmann classification of skeletal class 2 division 1. The patient was in stage 7 of fishmans classification (Figure 8B). The patient had orthognathic maxilla and retrognthic mandible with proclined upper and lower incisors. His lower anterior facial height was decreased. The upper posterior, lower anterior and lower posterior dentoalveolar height were also found to be reduced.

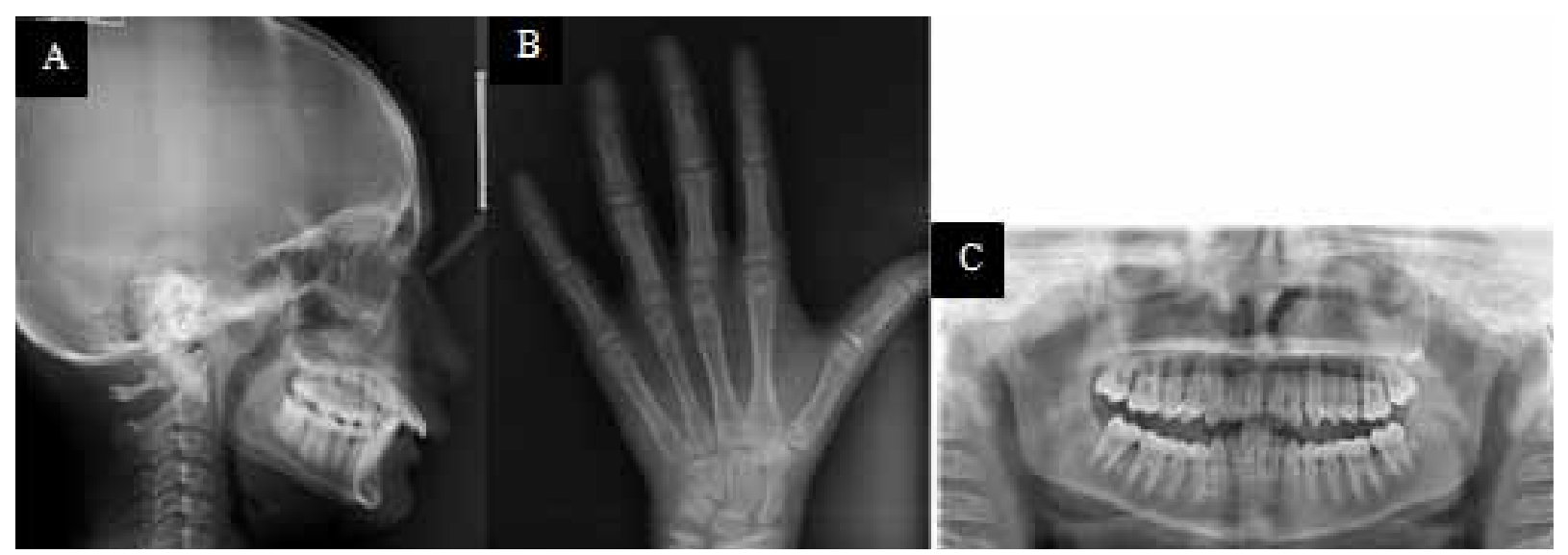

Figure 8: Pre-treatment radiographs A) Lateral cephalogram B) Hand wrist C) OPG

Pre treatment orthopantomogram (OPG) revealed that the patient had full permanent dentition with no missing or supernumerary teeth (Figure $8 \mathrm{C}$ ).

Based on the above findings, the patient was diagnosed as Angle's class II Division 1 dentoalveolar malocclusion on a class II skeletal base attributing to an orthognathic maxilla and retrognathic mandible on a low mandibular plane angle with deep bite, rotated 16,26 , spacing in upper anteriors with crowding in lower anteriors, proclined upper and lower anteriors with increased overjet.
Treatment Progress: A full orthodontic appliance of 0.022 slot MBT system was bonded first in the upper arch and later in the lower arch. Aligning and levelling was done sequentially with $0.016 \mathrm{NiTi}$ and $16 \times 22$ NiTi. After the completion of levelling and aligning, advansync2 appliance was assembled (Figure 9). The advansync 2 appliance was activated for $3 \mathrm{~mm}$ thrice during the therapy. At the end of the fixed functional therapy, bilateral class I molar and class I canine was obtained (Figure $10 \& 11$ ). The archwires were in 19 X25 $\mathrm{NiTi}$ at the end of the functional therapy.

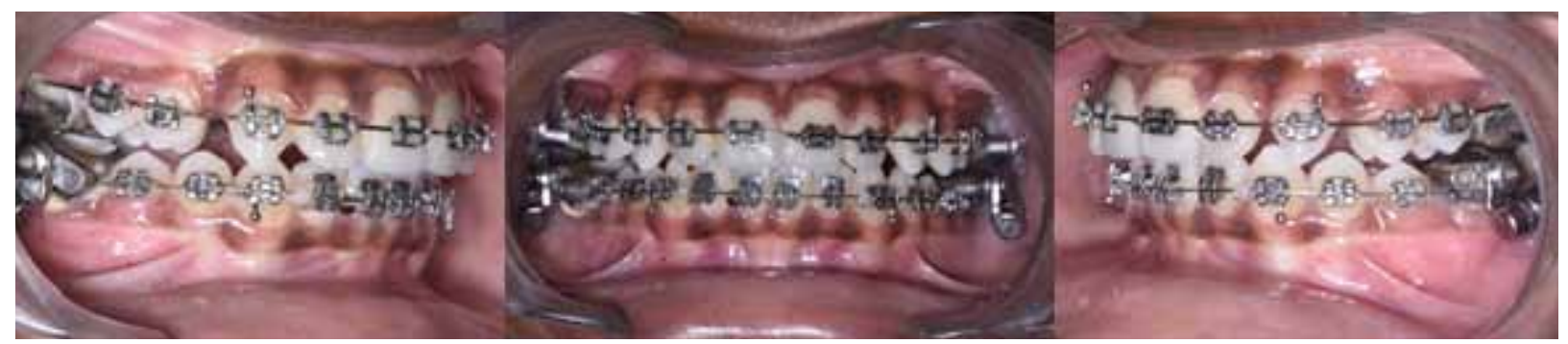

Figure 9: Advansync2 Fixation 

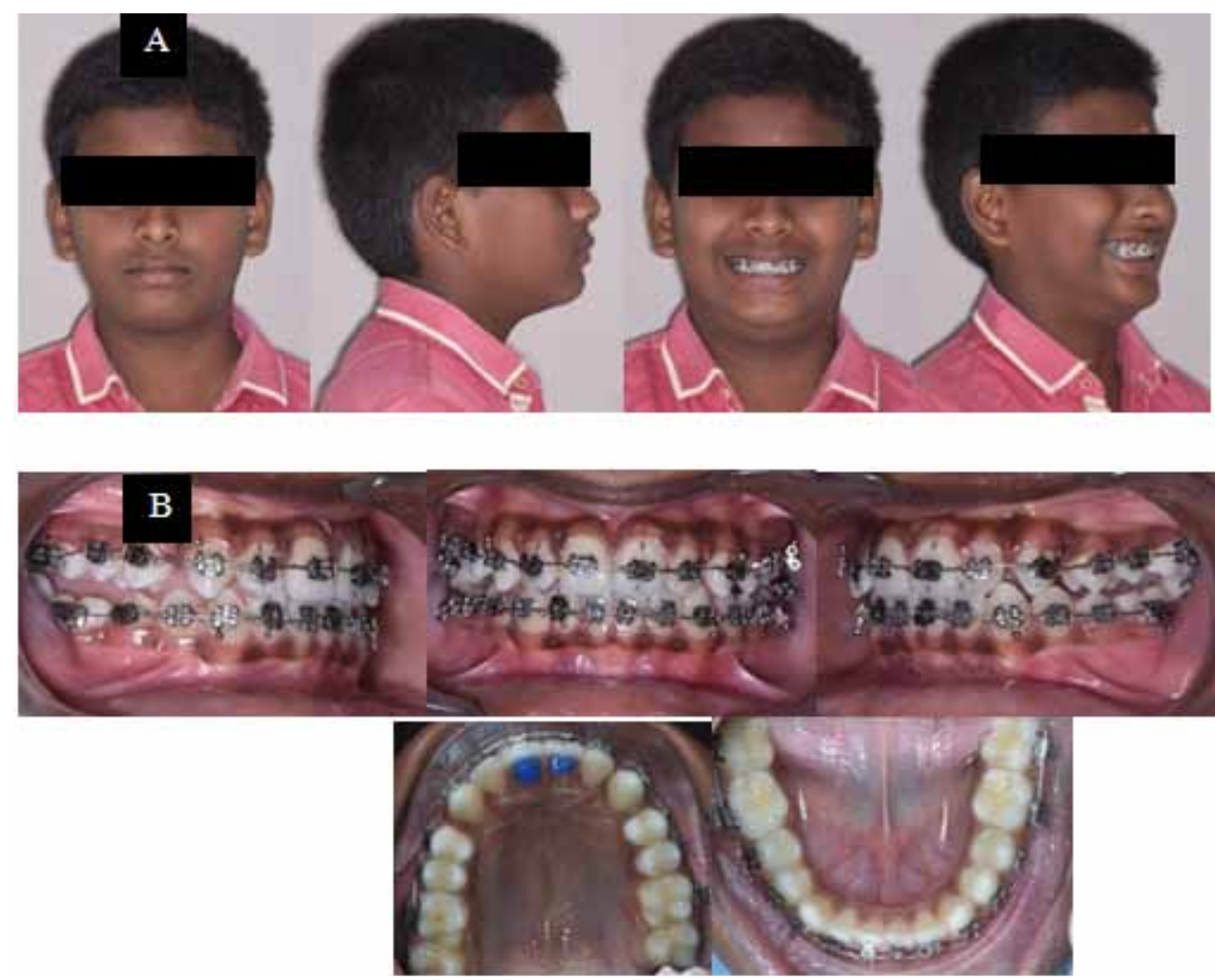

Figure 10: Post-functional A) Extra-oral B) Intra-oral photographs

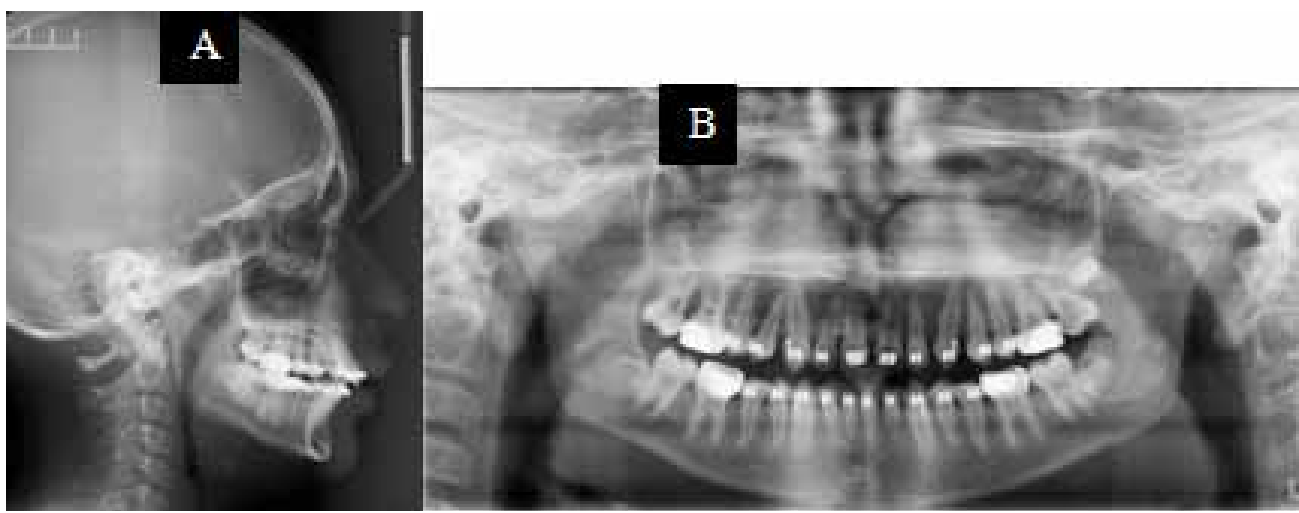

Figure 11: Post-functional radiographs A) Lateral cephalogram B) OPG

Treatment Results: The total duration of the treatment was 15 months. Post treatment photographs depicts the amount of intra oral and extra-oral changes obtained at the end of the treatment (Figure 12). The patient had obtained a pleasing smile and an esthetic soft tissue profile. The post treatment radiographs implies that an ideal inclination and angulation of the crown and root of all teeth have been obtained (Figure 13).There was an overall increase of about $3 \mathrm{~mm}$ in the maxillary inter-canine, inter-premolar and inter-molar widths suggesting arch expansion. Beggs wrap around retainer was delivered for the upper arch and a bonded lingual retainer was fixed in the lower arch. 

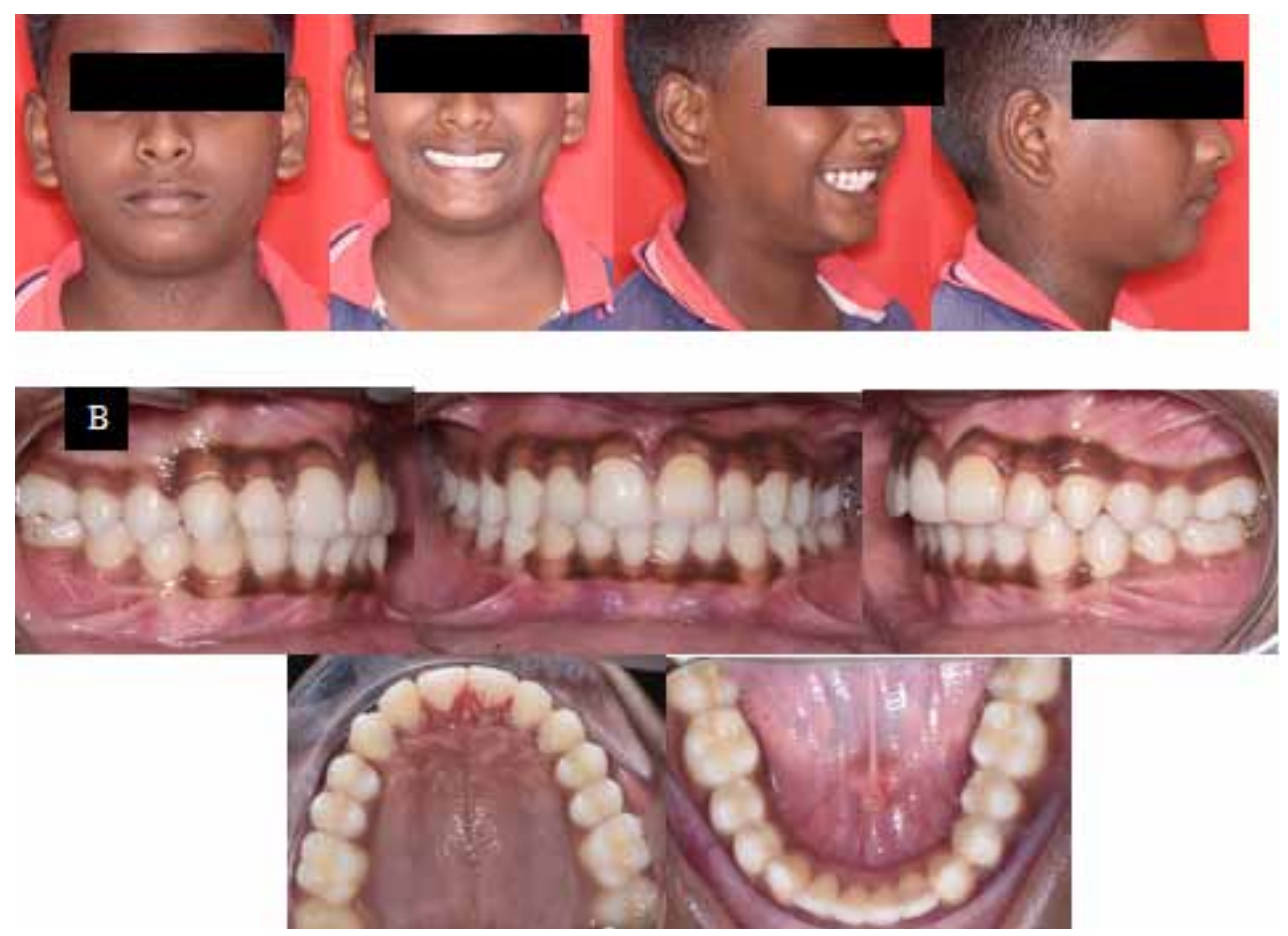

Figure 12: Post-treatment A) Extra-oral B) Intra-oral photographs

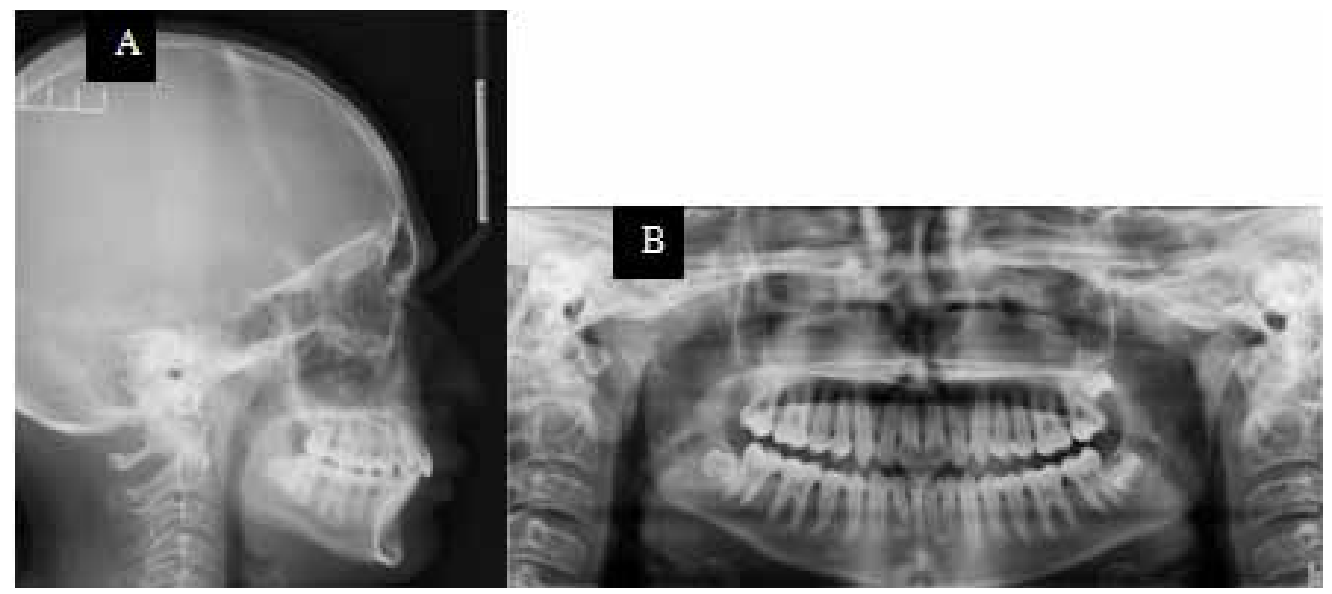

Figure 13: Post-treatment radiographs A) Lateral cephalogram B) OPG

\section{Discussion}

Different treatment modalities have been developed for the treatment of class II malocclusions such as selective extractions, orthopaedic treatment using head gear or functional appliances, removable and fixed inter or intra arch appliances and orthognathic surgeries. When the functional appliances are used during active growth periods (before or during puberty), they are intended to induce maximum skeletal growth. However, the amount of skeletal or dental change which will be obtained is difficult to quantify as it depends on various intrinsic and extrinsic factors.
Pangrazio et al stated that removable or fixed functional appliance bring about sagittal and vertical skeletal changes in the jaw positions resulting in orthopaedic and orthodontic changes ${ }^{[7]}$.Skeletal discrepancy correction during growth period can be achieved using removable as well as fixed functional appliance.

The AdvanSync2 appliance is a fixed functional appliance wherein there is no necessity to align and level the arches prior to the placement of the advansync 2 appliance, so capitalizing on residual growth is possible, with favourable decrease in the treatment duration. It 
is activated in increments until an edge to edge incisor relationship is attained as suggested by Dischinger ${ }^{[8]}$. The appliance can be removed after the overcorrection of sagittal discrepancy. As given by Jayachandran et al,Advan sync allowed for the simultaneous use of conventional edgewise appliance $(0.022 \times 0.028$ inch slots) ${ }^{[2]}$. It enhances the mandibular growth by constantly posturing the mandible forward upon closure through the telescoping mechanism.

The molar-to-molar attachment brings about intrusion of the molars and also mild proclination of the lower incisors. However, the amount of lower incisor proclination is lesser when compared to other fixed funtional appliances where the attachment is fixed to the mandibular anterior segment thereby resulting in greater proclination of the lower anteriors. The direction of the forces generated by the advansync 2 appliance includes sagittal, intrusive, and expansive vectors. The sagittal force vector has produced distal movement of the upper molars and also exerted an anterior force to the mandibular dentition and the mandible. Additionally, an intrusive force of the maxillary posterior region and mandibular anterior region was also evidenced. The push force generated by the appliance also leads to $2-3 \mathrm{~mm}$ of expansion of the maxillary dental arch. This can be evidenced by the increase in the maxillary intercanine, inter-premolar and the inter-molar widths in both our patients wherein there was an overall arch expansion of $3-4 \mathrm{~mm}$.

According to ruf and pancherz, Advan sync has produced significant mandibular growth changes in post pubertal patients, past their peak height velocities ${ }^{[9]}$. This can be evidenced by the post treatment cephalometric findings in both the patients. Al jewair et al stated that Advansync2 has a head gear like effect on maxillary molars and prevents their mesial movement which brings about class I molar relationship ${ }^{[6]}$. It also restrains the growth of maxilla which is beneficial in maxillary prognathic cases. This can be evidenced by the decrease in the 6 to ptv measurements on comparison of the pretreatment and post-treatment cephalograms. Maxillary growth restriction with combination of mandibular molar mesialization and mild lower incisor proclination (IMPA- $\left.108^{0} ; 99^{0}\right)$ with increase in FMA angle $\left(25^{0} ; 28^{0}\right)$ and lower anterior facial height (ANS-Gn $-58^{0} ; 62^{0}$ ) have also been evidenced [Table 1 and Table 2].

In cases having residual growth, alignment of the arches using stainless steel or TMA wire with incorporation of labial root torque prior to appliance placement will be beneficial and hence this has been done in case 2 to minimise the lower incisor proclination. Gandedkar and celikoglu et al, suggested that lower incisor proclination, which is a drawback of advansync2 appliance can be corrected by fixed appliance with labial root torque, cinch backs and heavy stabilizing wires with miniscrew anchorage ${ }^{[10,11]}$. By employing labial root torque, reduced proclination of the lower anteriors had been encountered in our second case.

Dislodgement of the bands from the molars were encountered. The bulkiness of the molar bands was a disadvantage which could not resist the occlusal forces resulting in dislodgement. Hence, re-cementation of the bands had to be employed. Maxillary and mandibular molars were intruded due to the occlusal forces and the molar-to-molar attachment. This can be evidenced in the post functional cephalogram wherein there was a decrease in the FMA and lower anterior facial height. This had to be counteracted by extruding the molars during the finishing and settling stages. Some amount of spacing had also occurred in the maxillary arch during the treatment which could be attributed to the arch expansion. This was however controlled using stabilising wires and cinch backs.

According to Bock et al 2015,there was a good dentoskeletal stability for Class II correction using Herbst appliance $^{[12]}$. According to Wigal et al, overcorrection using herbst appliance during early mixed dentition resulted in a significant reduction in overjet and correction of the molar relationship and also the correction was maintained after the fixed appliance therapy ${ }^{[13]}$. This was attributed to continuous restraint of the maxillary growth and the dentoalveolar adaptations. Since the advansync2 appliance is a miniaturised modification of the original Herbst appliance, the treatment stability can be expected to be the same.

\section{Conclusion}

The advansync2 appliance is an effective class II corrector producing an overall arch expansion of about $3-4 \mathrm{~mm}$ in the maxillary dental arch. The FMA and the lower anterior facial height had reduced postfuntionally (case $1-23^{0}$ and case $2-21^{\circ}$ ). However, due to the post functional extrusion of the molars, there was an increase in the FMA and the lower anterior facial height (case 1 $28^{0}$ and case $2-25^{\circ}$ ) at the end of the treatment. Use of fixed appliances with cinch backs and heavy stabilising 
wires prior to appliance fixation had reduced the lower incisor proclination. This case series highlights the use of the Advansync2 appliance as an efficient fixed functional appliance producing clinically significant restriction of the maxillary arch (case $1-3^{0}$ and case 2 $4^{0}$ reduction in ANB) confirming a short term orthopedic effect on the maxilla. The total treatment duration was shortened to15-18 months with simultaneous fixed appliance therapy.

\section{Ethical Clearance: Nil}

Source of Funding: Meenakshi Academy of Higher Education and Research, Chennai, India

Conflict of Interest: Nil

\section{References}

1. Singh G, Goyal V, Rastogi S, Menon AG, Aziz $\mathrm{SB}$, Sokhi RK. Management of class II division 1 malocclusion using fixed functional appliances: A case series. Journal of Indian Orthodontic Society. 2018 Mar;52(1):60-5.

2. Jayachandran S, Wiltshire WA, Hayasaki SM, Pinheiro FH. Comparison of AdvanSync and intermaxillary elastics in the correction of Class II malocclusions: A retrospective clinical study. American Journal of Orthodontics and Dentofacial Orthopedics. 2016 Dec 1;150(6):979-88.

3. Thushar BK, Verma S. Treatment Outcomes in the Sagittal and Vertical Dimensions with the AdvanSync2 Class II Corrector-A Case Series. Journal of Contemporary Orthodontics. 2018 Jul;2(3):14-26.

4. Advan sync 2 fixed functional class 2 corrector advanced age, case report, Mathur et al, HTAJOCD 2019

5. Pancherz H. The Herbst appliance-its biologic effects and clinical use. American Journal of Orthodontics. 1985 Jan 1;87(1):1-20.
6. Al-Jewair TS, Preston CB, Moll EM, Dischinger T. A comparison of the MARA and the AdvanSync functional appliances in the treatment of Class II malocclusion. The Angle Orthodontist. 2012 Sep;82(5):907-14.

7. Pangrazio-Kulbersh V, Berger JL, Chermak DS, Kaczynski R, Simon ES, Haerian A. Treatment effects of the mandibular anterior repositioning appliance on patients with Class II malocclusion. American journal of orthodontics and dentofacial orthopedics. 2003 Mar 1;123(3):286-95.

8. Dischinger BM. Skeletal class II case presentation: Utilization of the advansync 2 appliance. APOS Trends in Orthodontics. 2018;8(3):168-74.

9. Ruf S, Pancherz H. Herbst/multibracket appliance treatment of Class II division 1 malocclusions in early and late adulthood. A prospective cephalometric study of consecutively treated subjects. The European Journal of Orthodontics. 2006 Aug 1;28(4):352-60.

10. Gandedkar NH, Revankar AV, Ganeshkar SV. Correction of a severe skeletal Class II occlusion with a fixed functional appliance anchored on mini-implants: a patient report. World journal of orthodontics. 2010 Dec 1;11(4).

11. Celikoglu M, Unal T, Bayram M, Candirli C. Treatment of a skeletal Class II malocclusion using fixed functional appliance with miniplate anchorage. European journal of dentistry. 2014 Apr;8(2):276.

12. Bock NC, Ruf S. Dentoskeletal changes in adult Class II division 1 Herbst treatment - how much is left after the retention period?. European journal of orthodontics. 2012 Dec 1;34(6):747-53.

13. Wigal TG, Dischinger $\mathrm{T}$, Martin $\mathrm{C}$, Razmus $\mathrm{T}$, Gunel E, Ngan P. Stability of Class II treatment with an edgewise crowned Herbst appliance in the early mixed dentition: Skeletal and dental changes. American journal of orthodontics and dentofacial orthopedics. 2011 Aug 1;140(2):210-23. 